\title{
EL DESEMPEÑO DE ÍCONOS COMO HERRAMIENTA GRÁFICA PARA COMUNICAR LA EMERGENCIA
}

\author{
Rodrigo Ramírez M. ${ }^{1,2}$ *
}

\section{RESUMEN}

La experiencia humana es un foco del Diseño, transferible a escenarios como el de la comunicación del riesgo. Una emergencia altera radicalmente la experiencia de información, considerada un soporte estructural para las acciones en contextos críticos. El diseño de información ha sido definido como una combinación de arte y ciencia de presentar información para ser usada eficiente y efectivamente por humanos. La información bien diseñada permite a las personas visualizar, comprender mensajes y facilitar decisiones. Cotidianamente los símbolos estandarizados o íconos constituyen una solución típica, ya que simplifican el contenido permitiendo sortear barreras de acceso. Se presenta el proyecto Guemil, una iniciativa de íconos para representar conceptualmente el ciclo Antes > Durante > Después de una emergencia. El set cubre la experiencia completa en el ciclo de la Gestión del Riesgo de Desastres (GRD). Buscando entender la eficacia como solución gráfica supuestamente universal, se exploró sobre los significados y diferencias que las personas asignan a cada ícono. Su desempeño se midió desde pruebas estandarizadas y aplicables internacionalmente. Desde la evidencia obtenida se revisan aspectos específicos, analizando el desempeño en casos y discutiendo nociones sobre la comprensión de información en emergencia. Enfocado en preparación y adopción, se concluye reflexionando sobre oportunidades futuras del proyecto.

\section{PALABRAS CLAVE}

Comunicación, Emergencia, Íconos, Significados, Preparación

\section{MEASURING PERFORMANCE OF ICONS AS A VISUAL TOOL TO COMMUNICATE EMERGENCIES}

\section{ABSTRACT}

Human experience is a focus of Design, transferrable to scenarios such as risk communication. An emergency experience radically affects the interaction with information, transforming this in a structural support for action in critical contexts. Information Design has been defined as a combination of art and science to present information to be used efficiently and effectively by humans. An optimal designed information allows people to see, understand messages and it facilitates decisions. In everyday, standardized symbols -or icons, constitute a typical solution, as they simplify contents allowing to deal with access barriers. This paper introduces Guemil Project, an icon initiative oriented to conceptually represent the cycle Before > During > After an Emergency. The set is applicable to the whole cycle of Disaster Risk Management (DRM). Oriented to know about effectiveness of such a language supposedly universal, a key aim is to raise what meaning and differences users can assign to their visual design. To measure its performance, systematic tests were developed and applied internationally. From evidence, specific aspects are reviewed, analyzing cases that exhibits their performance and contributing to establish notions about comprehension of information in emergency. It concludes reflecting about future opportunities, focused on preparation and adoption.

\section{KEYWORDS}

Communication, Emergency, Icons, Meaning, Preparedness
1. Escuela de Diseño, Facultad de Arquitectura, Diseño y Estudios Urbanos, Pontificia Universidad Católica de Chile, Chile

2. School of Design, Hong Kong Polytechnic University, China

*Autor de correspondencia: rramireo@uc.cl

\section{RECIBIDO}

18 de septiembre de 2017

\section{ACEPTADO}

20 de noviembre de 2017

\section{PUBLICADO}

30 de enero de 2018

\section{Formato cita}

Recomendada (APA): Ramirez M., R. (2018). El desempeño de íconos como herramienta gráfica para comunicar la emergencia. REDER, 2(1), pp.71-87.

\section{(c) (i) (8)}

Todos los artículos publicados en REDER siguen una política de Acceso Abierto y se respaldan en una Licencia CreativeCommons Atribución-NoComercial 4.0 Internacional.

\section{Revista de Estudios}

Latinoamericanos sobre Reducción del Riesgo de Desastres (REDER) 


\section{DEFINICIONES INTRODUCTORIAS}

El artículo busca centrarse en aspectos específicos del ciclo del riesgo de desastres, que en este caso es relacionado con la experiencia de las personas y la necesidad de información. Es por ello que se parte con algunas definiciones introductorias:

\section{El Manejo del Riesgo de Desastres}

El riesgo de desastre es la potencial pérdida o destrucción total o parcial de vidas o recursos que puede ocurrir a un sistema, sociedad o comunidad en un periodo específico de tiempo (UNISDR, 2015). Este puede determinarse por la combinación de factores asociados a: (1) Amenaza; (2) Exposición; (3) Vulnerabilidad.

La Gestión del Riesgo de Desastres (GRD) es conocida usualmente por su nombre y acrónimo en inglés (Disaster Risk Management, DRM). Es un proceso continuo definido por la UNISDR (2017) como un plan que define 'las metas, objetivos específicos y acciones para reducir el riesgo de desastre'. Considera una serie de actividades para la coordinación, recursos y desarrollo, promovidos desde el Marco de Acción de Sendai para la Reducción del Riesgo de Desastre, 2015-2030, conocido como el 'Sendai Framework'. Este manejo planificado contribuye a que las comunidades puedan reconocer su vulnerabilidad a las amenazas y enfrentar los eventuales desastres. En este proceso secuencial hay dos partes que resultan fundamentales: (1) La preparación previa a la emergencia o desastre, y (2) la recuperación posterior a la disrupción del desastre. En EE. UU. la Agencia Federal para el Manejo de Emergencias (Federal Emergency Management Agency, FEMA) en su Marco Nacional para la Recuperación de Desastres (en inglés NDRF, 2009) define que la recuperación 'comienza siempre con una preparación previa al desastre'. También, reconoce que la recuperación transcurre de forma continua en el ciclo del riesgo. El modelo conocido como Ciclo de Gestión del Riesgo de Desastres (DRMC), como el desarrollado por TorqAid (2016) y que sintetiza la Figura 1 respecto al original, presenta este proceso como una secuencia temporal de eventos: Antes, durante, después de una emergencia.

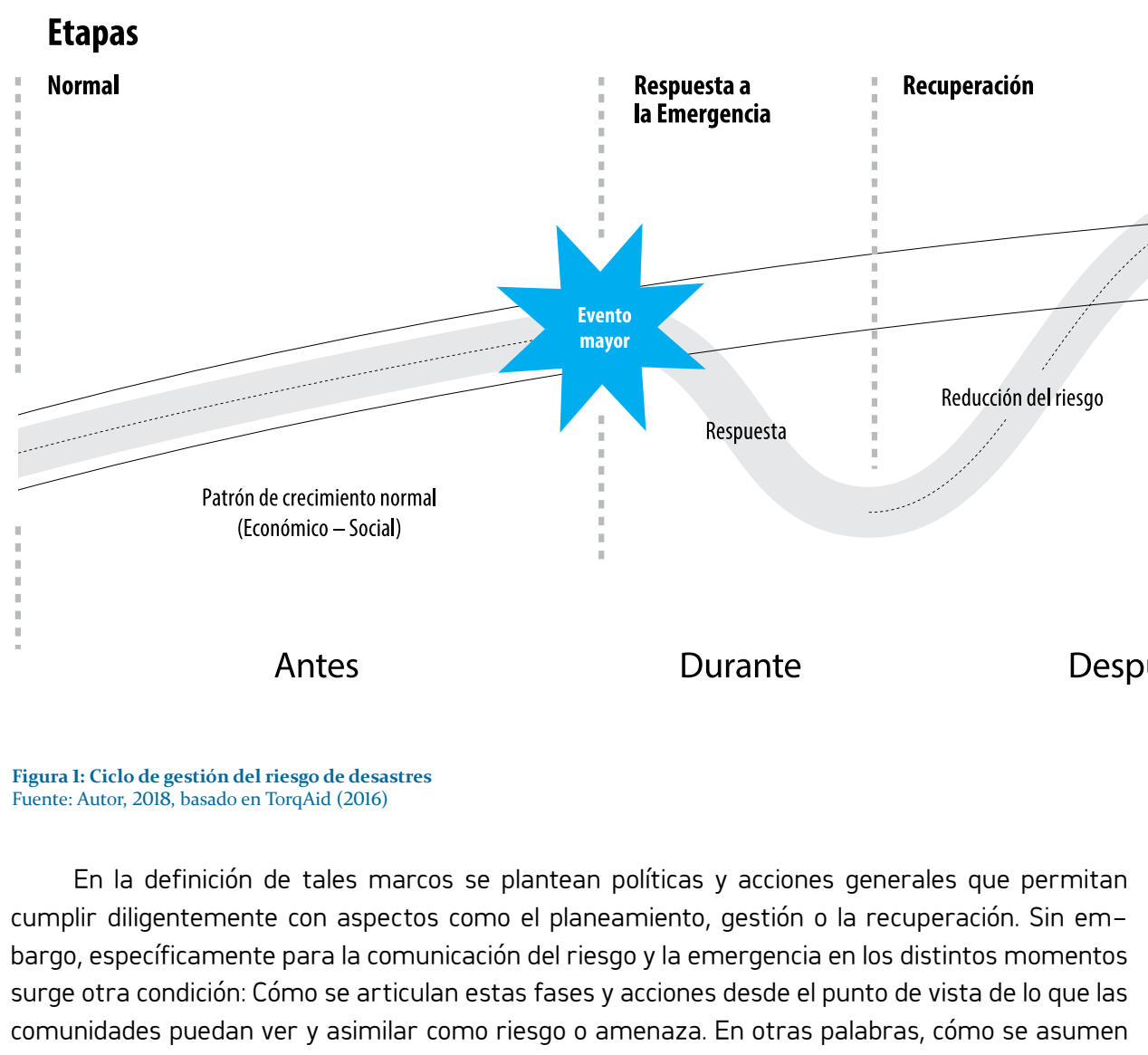


acciones centradas en lo que las personas y sus organizaciones puedan enfrentar. De esta forma, es necesario definir qué es la llamada experiencia de usuario.

\section{La experiencia de las personas y su diseño}

Como disciplina centrada en el ser humano, ejercicios como la empatía, el observar interacciones humanas resultan naturales para el diseño. Tal como ocurre con categorizaciones según el ámbito en que se desenvuelven (gobierno = ciudadanos $/$ mercado $=$ consumidores) las personas suelen ser etiquetadas como 'usuarios' al momento de utilizar el diseño de un producto o servicio; de ahí la extensión de términos como 'estudios de usuario' o 'usabilidad'. Así, la Organización Internacional de Estandarización, ISO (2009) define la Experiencia de Usuario como una vivencia donde emergen "las percepciones y respuestas que resultan del uso o la intención de uso de un producto, sistema o servicio". Agrega que ello involucra las emociones, creencias, preferencias, percepciones, respuestas físicas y sicológicas, sus comportamientos y resultados que puedan ocurrir antes, durante y después de que transcurren. En paralelo, Nielsen y Norman (2017), establecen como primer requerimiento para una experiencia ejemplar, el que se conozcan "exactamente las necesidades del usuario".

Trascendiendo de los paradigmas por los que se ha conocido al diseño en la sociedad industrial, hoy una combinación de investigación + capacidades proyectuales, constituye un corpus de conocimiento disciplinar que puede ser integrado a otros campos como las ciencias, la gestión, o la tecnología (Cross, 2001), aportando a lo que se denomina genéricamente como 'factores humanos'. En los procesos del diseño centrado en los usuarios, se parte levantando información desde aquello que las personas hacen, viven, o sueñan buscando abarcar sus necesidades, para luego desarrollar creativa y técnicamente nuevos productos, experiencias, o combinaciones. Tal como lo indica Norman (2004), un factor humano como la emoción es central para entender lo que como personas preferimos o rechazamos. En sus palabras, "aquellos productos [o experiencias] que son una dicha de tener [o vivir]". Por otro lado, un diseño mal resuelto representa por lo general una experiencia evitable; todas estas aproximaciones 'viscerales' que moldean luego los comportamientos y las decisiones 'reflexivas' (Norman, 2004).

Al definir características del diseño, Wasson (2000) considera un producto "exitoso" aquel que es adoptado [por las personas]. Por lo tanto si un diseño se conecta a nivel emocional, satisface las necesidades del contexto y aprovecha los recursos disponibles, tiene mayores posibilidades de ser incorporado naturalmente en las prácticas cotidianas. Esto puede aplicarse también a la experiencia en situaciones de riesgo: Las carencias y emociones humanas son ámbitos prioritarios en el cual trabajar desde el diseño centrado en las personas, donde elementos como la información pueden ser centrales para optimizar la experiencia reduciendo la incertidumbre, o facilitando la toma de decisiones.

\section{Información como parte de la experiencia}

Uno de los aspectos clave en cualquier experiencia humana es el de la información. De acuerdo a Horn, (1999), la disciplina del Diseño de Información es definida como "el arte y ciencia de presentar información para que pueda ser usada por humanos con eficiencia y efectividad". Por sus alcances, se trata también de un campo multi-dimensional donde convergen diversas disciplinas en su aplicación, que en la llamada era de la información forma parte central de lo cotidiano para personas y organizaciones (Pettersson, 2002). Para la articulación de una comunicación efectiva, esta combinación dual de Arte + Ciencia, es abordable desde múltiples perspectivas, como ejemplos: Hacer visibles los mensajes, facilitar el acceso y comprensión de grandes cantidades de información, o establecer su efectividad observando qué se hace respecto a cómo se presenta, todas acciones que pueden ser al mismo tiempo altamente creativas y medibles en su desempeño. La Figura 2, basada en el trabajo de Allard et al. (2014) para el diseño de información a usuarios del transporte, sintetiza este ciclo de la información respecto a la experiencia.

Tal como lo define Frascara (2014; 2011), diseñar la información no es solo un aspecto de configuración gráfica: implica principalmente entender qué contenido requieren las personas en un contexto específico, de esta forma generar mensajes significativos. Más allá de solo visualizar, las necesidades de información de las personas pueden ser cubiertas relacionándolas estrechamente con su experiencia. De esta forma, se vuelve clave cómo estén estructurados los mensajes 
respecto al contenido y su configuración. Así, un diseño adecuado permitirá también modelar la experiencia colectiva, facilitando el ver, entender y actuar en situaciones específicas.

$\begin{array}{llll}\text { Visualizar } & \text { Entender } & \text { Aplicar } \\ & \text { Quées? } & \text { Cómo proceder? } & \\ \begin{array}{l}\text { Diseñar para } \\ \text { hacer visible }\end{array} & & \begin{array}{l}\text { Diseñar para hacer } \\ \text { comprensible }\end{array} & \begin{array}{l}\text { Diseñar para la } \\ \text { acción / decisiones }\end{array}\end{array}$

Figura 2: Ver $>$ Entender $>$ Aplicar la información Fuente: Autor, 2018, basado en Allard et al. (2014)

\section{La comunicación del riesgo y la emergencia}

Tal como lo remarcan Bui \& Sebastian (2011) citando a Van Someren, Netten et al. (2005), la información constituye un "soporte estructural para todas las actividades en condiciones extremas con alta incertidumbre". Debido a su naturaleza humana y alcance global, el manejo del riesgo y la emergencia surgen como uno de los mayores desafíos para el desarrollo (WEF, 2017), por lo que la comunicación efectiva en este ámbito es prioritaria. Como lo han abordado extensivamente Wogalter, Mayhorn et al. (1999; 2001; 2017) mensajes visuales oportunamente desplegados como las advertencias de seguridad, constituyen herramientas efectivas para asistir a las personas en sus actividades cotidianas. Sin embargo, una situación disruptiva altera radicalmente el interactuar con información. Como indica Jaenichen (2011), lo que podría resultar natural de comprender en lo cotidiano se transforma radicalmente en contextos extremos. En estas las condiciones para el procesamiento de información son otras: La normalidad es alterada y para las personas la necesidad de información -lo que requiere ver y entender para actuar- puede variar dramáticamente.

Quienes están expuestos a situaciones de riesgo o emergencia, requieren una calidad visual consistente en soportes de comunicación para operar en diversos momentos. Sin embargo, comúnmente se observa que los mensajes de información suelen estar dispersos en diversos tipos de lenguaje, y pensada más desde la indicación o el soporte para el momento mismo en que esta ocurre (señalización para evacuar, ver ejemplos en Figura 3) y menos desde un plan secuencial sobre qué tipo de representación puede ser más visible, qué contenido puede más entendible y cómo distribuir eficientemente el contenido en múltiples soportes.
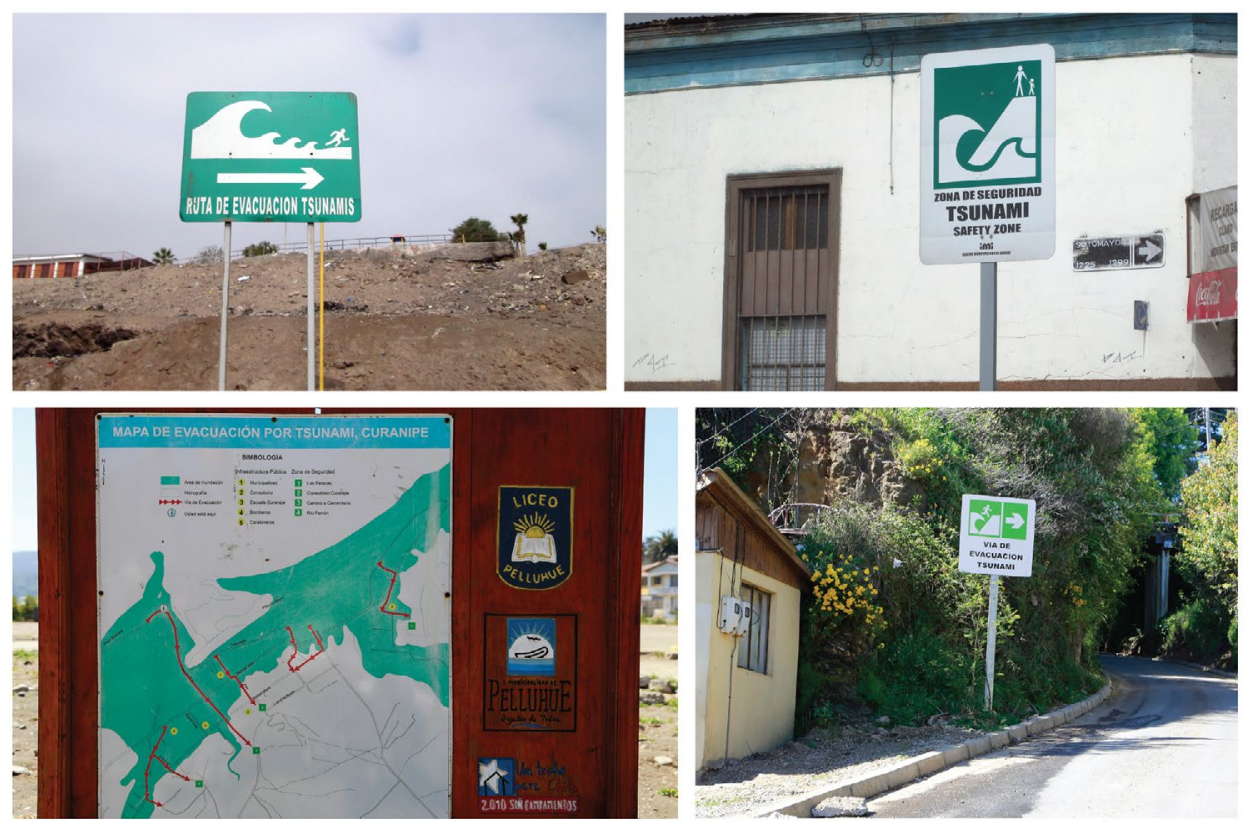

Figura 3: Ejemplos de inconsistencia visual en soportes de información de emergencia, Chile Fuente: Archivo CIGIDEN, 2015 
Sin embargo, puede haber grandes diferencias entre el ver, entender y luego aplicar la información, las que pueden resultar dramáticas ante una disrupción de la normalidad, en el momento mismo de la emergencia o a posterior. Por ello se vuelve importante este cruce entre experiencia y GRD, para enfocar los esfuerzos de información hacia elementos reconocibles, simples de entender y que se desplieguen en todas las etapas del riesgo, aprovechando por ejemplo una motivación mayor en etapas previas. Asumir cada una respecto a estas necesidades podría estar más en sintonía con la experiencia de las personas y medir el desempeño de la comunicación. La Figura 4 esquematiza un modelo, mostrando cómo los mensajes pueden distribuirse como parte de una continuidad, que cruce los objetivos en el Riesgo, la Experiencia y la Información.

\begin{tabular}{|c|c|c|c|c|c|}
\hline Riesgo & Preparación & & Mitigación & Acción & \\
\hline Experiencia & Antes & & Durante & & Después \\
\hline \multirow[t]{4}{*}{ Información } & Visualizar & Entender & $>$ & Aplicar & \\
\hline & Riesgos & Amenazas & Disrupción & Reacción & Recuperación \\
\hline & Identificación & Procedimientos & Acciones & Reporte & Reconstrucción \\
\hline & $\begin{array}{l}\text { Preparación } \\
\text { Difusión }\end{array}$ & Aprendizaje & Decisiones & Asistencia & \\
\hline
\end{tabular}

Figura 4: La GRD desde el foco de la experiencia de las personas e información para Visualizar > Entender > Aplicar Fuente: Autor, 2018

Entender las necesidades que se generan ante escenarios disruptivos, visibilizando lo que debiese ser prioritario, qué evitar hacer, cómo prepararse ante un evento potencial o mitigar en un desastre, podría contribuir una mejor preparación reduciendo la incertidumbre y aportando a la conformación de una cultura resiliente. En esto el diseño de información constituye un aporte sustancial, facilitando la comunicación del riesgo de múltiples formas, soportes y manteniendo un soporte continuo en la experiencia. Adicionalmente, siguiendo el foco centrado en las personas, el diseño de información puede contribuir a comunicar cómo prepararse, entendiendo mejor qué ocurre -y cómo proceder - en las distintas fases de la GRD. Dispositivos de información visual en la vida cotidiana ejemplifican el aprendizaje permanente que constituye su uso cotidiano, denominado por Mijksenaar y Westendorp (2001), como "el test diario de inteligencia visual".

Tal como se ha indicado extensivamente (Parsons et al.. 1999, Wogalter et al., 1999, Marom \& Goldschmidt, 2011), la implementación de herramientas gráficas puede contribuir a mejorar la experiencia al facilitar el acceso a información visible sobre el riesgo, la que además puede ser comprensible y traducible a acciones efectivas que promuevan conductas seguras, bajo un lenguaje universal. El campo de desarrollo es amplio y abierto al cruce de disciplinas.

\section{Otros casos de comunicación del riesgo a través de herramientas gráficas}

Complementando los antecedentes presentados, algunos casos internacionales brevemente comentados permiten ejemplificar mejor el enfoque de comunicación para el riesgo basado en la experiencia de las personas. La Figura 5 a continuación los ilustra. 


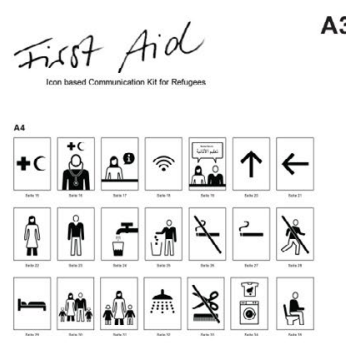

A3
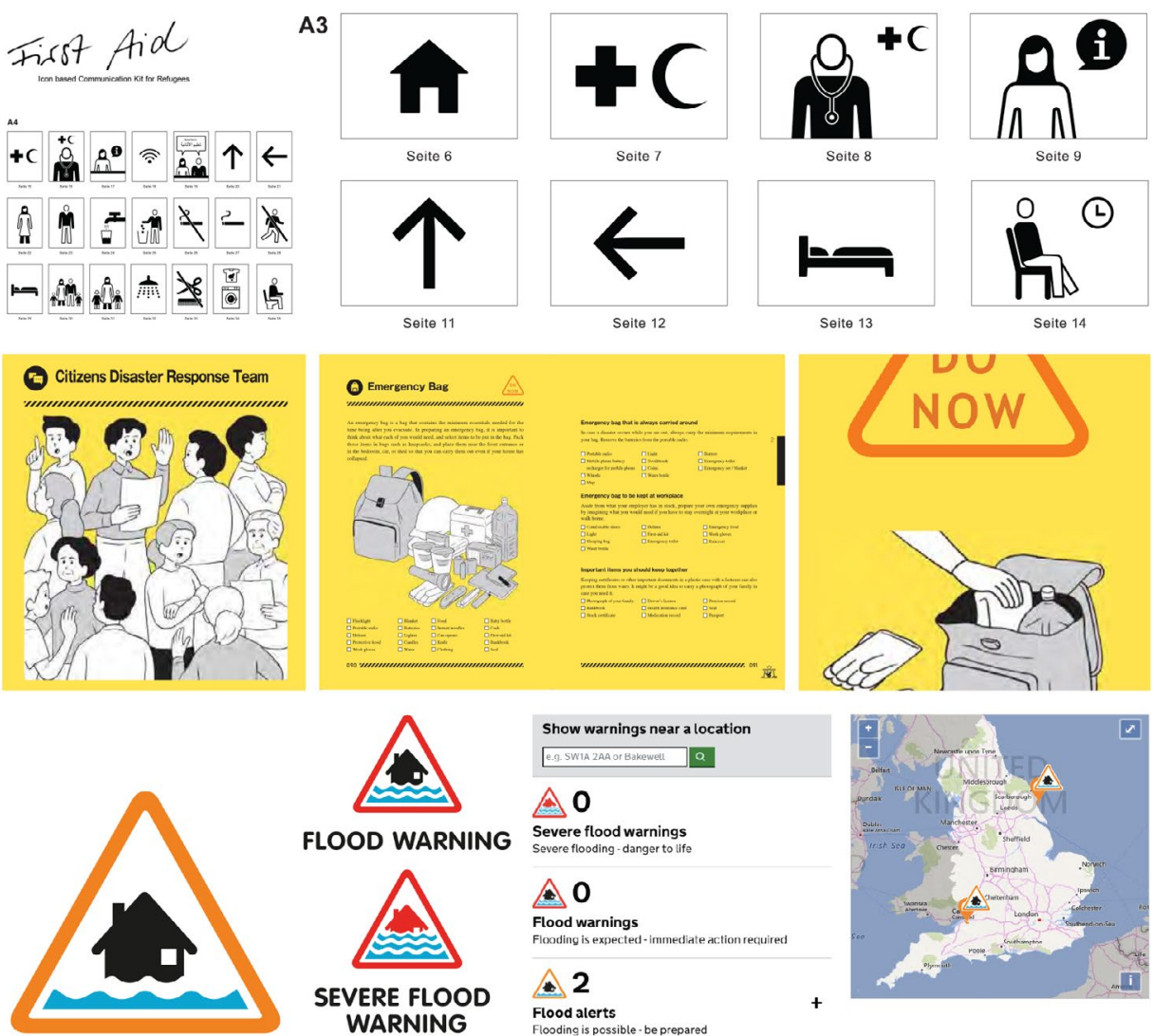

FLOOD WARNING

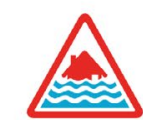

SEVERE FLOOD

WARNING
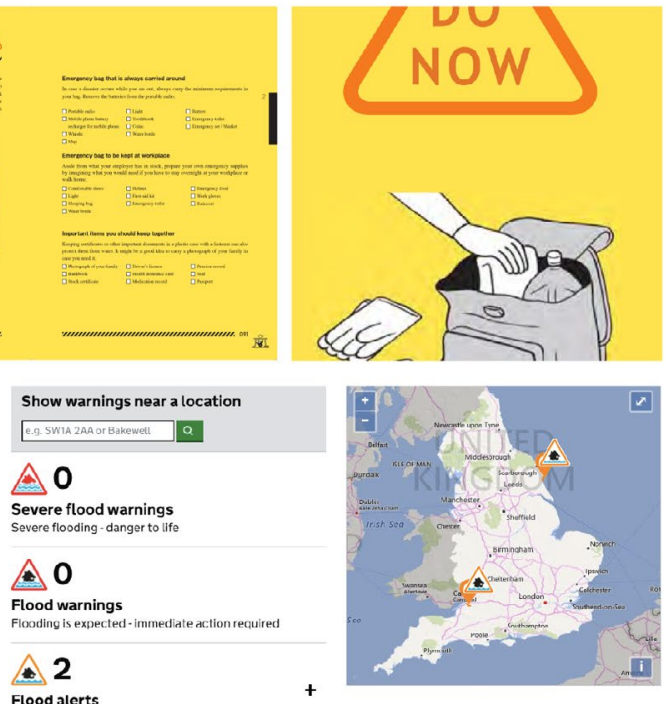

Figura 5: First Aid (arriba), Tokyo Bousai (medio), Flood Warnings (abajo). Tres ejemplos de comunicación para el riesgo a través de herramientas gráficas

- Información para entender (Qué es): El kit de comunicación 'First Aid' (arriba) para refugiados basado en íconos y soportes de comunicación multi-lenguaje (Austria, 2015). Este se enfoca en la ayuda humanitaria para desplazados y refugiados que arriban a Europa, representando necesidades de información que tengan, a través de soportes de bajo costo y formatos normalizados de fácil implementación.

- Información sobre procedimientos (Cómo hacer): La guía visual 'Tokio Bousai' (medio) para proceder ante una amenaza sísmica en Tokio, Japón. Este cubre diversos aspectos para la preparación, reacción y recuperación del sismo. La guía impresa -que incluye incluso una narración gráfica en formato manga- se distribuyó a todos los habitantes de la ciudad a fines de 2015.

- Información para el manejo (Cómo preparar): La iniciativa de información permanente 'Flood Warnings' (arriba)

impulsada por organizaciones gubernamentales y privadas en el Reino Unido, que busca que las personas conozcan y evalúen por si mismas el riesgo de inundación al que están expuestas. Se basa en elementos iconográficos y de color, que se repiten consistentemente en los soportes donde se publican.

Como se muestra, las herramientas gráficas pueden ser soportes para acceder a la información y optimizar la comunicación. Estas pueden contribuir a la experiencia humana en situaciones de riesgo, marcando diferencias entre estar preparado, a salvo o afectado. Las características pueden variar dependiendo de múltiples variables (tipo de amenaza, contexto cultural, familiaridad con lo visual). Aunque se puede observar una constante, que es un lenguaje gráfico consistente -normalizado-, donde el usuario puede reconocer, aprender, recordar y lo más importante: Hacer sentido desde su propia perspectiva sobre el mensaje desplegado, transfiriendo este a acciones (en algunos casos para ejecutar inmediatamente). La siguiente sección presenta el desarrollo de una iniciativa de íconos que comparte objetivos similares.

\section{ÍCONOS PARA COMUNICAR LA EMERGENCIA: EL PROYECTO GUEMIL \\ Normalizar el lenguaje para la emergencia}

Como se ha descrito, un diseño de información efectivo puede ayudar a la experiencia de las personas, permitiendo comunicar efectivamente en todas las etapas de la GRD a través de un lenguaje normalizado. En este caso se presenta un proyecto basado en uno de los paradigmas visuales de este lenguaje: el de los íconos, pictogramas o símbolos, denominaciones que son usadas 
indistintamente. Los íconos han sido definidos por Abdullah \& Hübner como elementos gráficos "portadores de significado" (2006).

El lenguaje de íconos tiene como fin desplegar unidades de información altamente visibles, recordables e idealmente entendibles, donde puedan verse representadas de forma simple elementos, situaciones y/o acciones. Estos se utilizan extensivamente para representar información de forma sintetizada, multiplataforma y entendible de modo rápido, asociado a menudo a las necesidades de usuarios en espacios públicos o al interactuar con tecnologías, constituyendo una lingua franca de nuestro tiempo (Zender \& Mejía, 2013; Ramírez, 2017).

\section{Qué es Guemil}

Guemil, del Mapudungun Ngümin, 'greca o símbolo' (Loncón, 2011), es una iniciativa basada en íconos para representar situaciones de riesgo y emergencia. En términos generales, se diseñó como un set de símbolos que abarca desde la representación icónica (analógica) a la simbólica (abstracta), en un lenguaje simple y de uso público. La Figura 6 grafica estas diferencias convencionales, y cómo el set incorpora en su diseño diversas tipologías de representación. Su concepto central es representar tres momentos de la experiencia a través de un estilo visual normalizado: Antes $>$ Durante $>$ después de una emergencia.

\section{Representación}

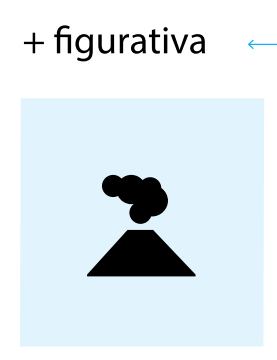

Ícono

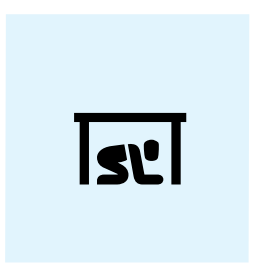

Pictograma
+ abstracta

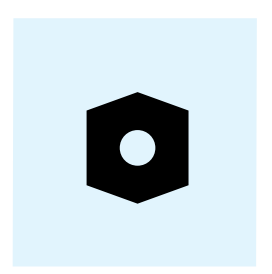

Símbolo

Figura 6: Tres diferencias fundamentales en la escala de representaciones de Guemil: ícono + pictograma + símbolo Fuente: Autor, 2018

\section{Cómo funciona}

De lo analógico a lo abstracto, Guemil dispone un conjunto de 96 pictogramas para representar conceptualmente el ciclo de riesgo completo: estos pueden representar personas, acciones, amenazas, situaciones entre otras, dependiendo del contexto y soporte en que se aplique. Para optimizar su funcionamiento, este conjunto ha sido 'empaquetado' en una fuente tipográfica open source -de licenciamiento Creative Commons y descarga abierta-, que puede ser instalada en computadores personales o servidores web. Para facilitarlo, se ha dispuesto una plataforma web que permite su acceso, descarga y testeo (www.guemil.info).

Los íconos pueden funcionar en múltiples escenarios, aunque básicamente con dos niveles de uso: Al emitir información visual simplificada y al requerir información de acceso rápido. De esta forma más allá de la simpleza y el acceso, otros fundamentos relevantes para abordar una comunicación del riesgo basada en íconos pueden ser varias: Su flexibilidad de adaptarse a requerimientos multi-culturales, con una aparente facilidad de aprendizaje; la facilidad para ser aplicado en múltiples soportes con una calidad consistente, complementando otros lenguajes como el texto; su relativo bajo costo al aplicarlo y también una aspiración a conformar un recurso de acceso universal para transmitir mensajes. La Figura 7, muestra como imagen ilustrativa algunos de los desarrollados en la versión 0.5b, 2016. 


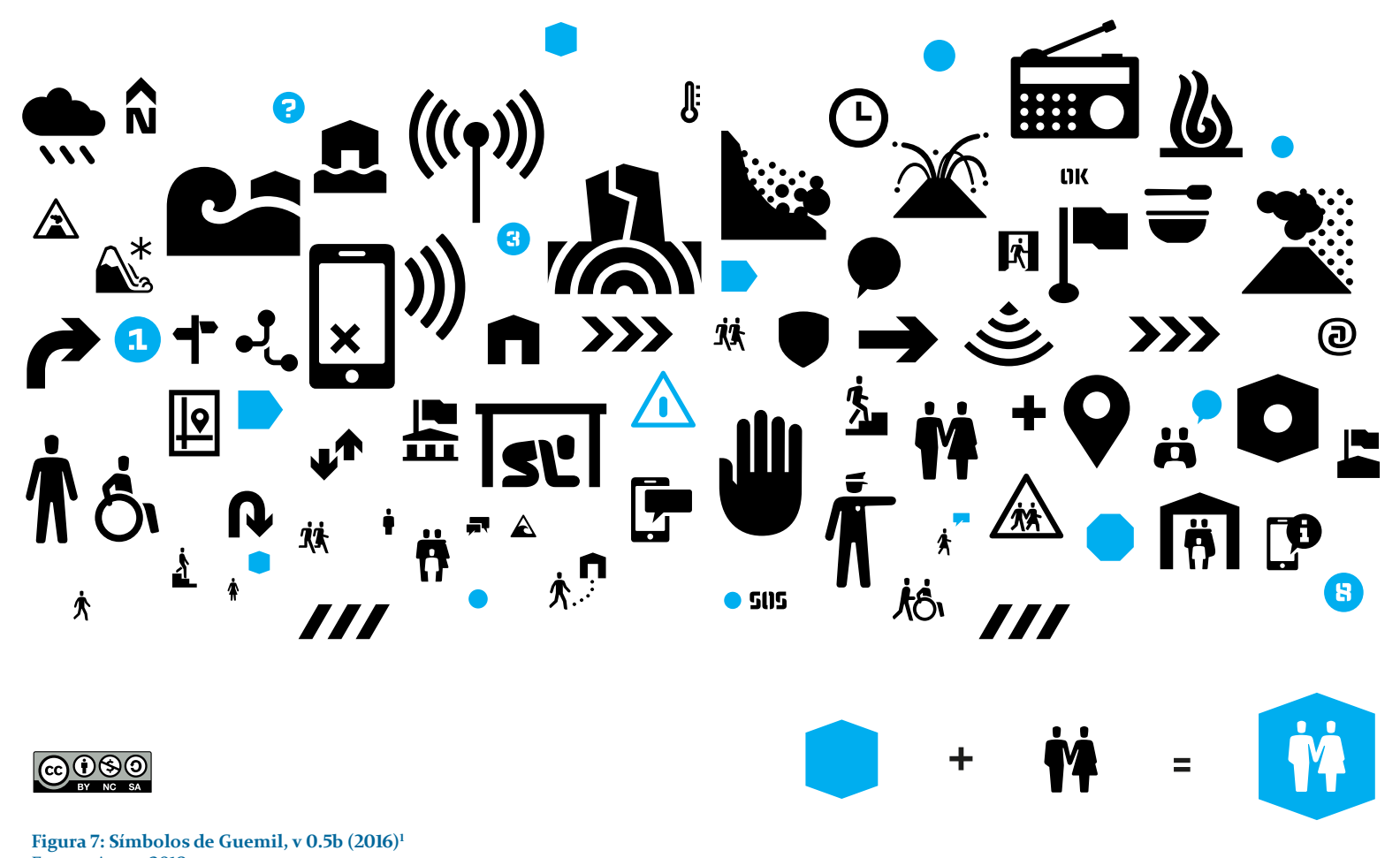

Fuente: Autor, 2018

\section{Dónde se aplica}

Básicamente, Guemil constituye un insumo base para la generación de otros soportes de comunicación. Los íconos pueden aplicarse como herramienta de información visual para situaciones o acciones donde sea necesario adaptarlas a diversos soportes, tecnologías y etapas de la GRD, permitiendo sortear barreras idiomáticas, tecnológicas y/o culturales. Constituye un recurso potencialmente efectivo para planear y desplegar comunicación puntual en las distintas fases de la emergencia, siendo consistente.

Innovando en la comunicación del riesgo, una decisión en su diseño fue aprovechar el ámbito digital y hacerlo accesible, facilitando su aplicación con lo que se fortalece una potencial adopción. De esta forma, puede implementarse para representar información relacionada por ejemplo con la preparación del riesgo, como soporte para la toma de decisiones y mitigación del desastre, o en acciones para la recuperación; su campo de aplicación es abierto y depende de otros factores que se explicitan más adelante.

Adicionalmente, uno de los aspectos más interesantes en el desarrollo del proyecto, es que ha sido un motivo para el desarrollo de investigación aplicada, en un en un cruce disciplinar y tópico prioritario. Guemil es uno de los proyectos fundadores del Design Network for Emergency Management (dnem.org), agrupación de profesionales, académicos e investigadores cuyo foco es contribuir al desarrollo interdisciplinar de herramientas de comunicación para la emergencia. En ello el diseño ha resultado ser un articulador, acercando disciplinas y permitiendo sumar en el campo de la investigación proyectual a través de iniciativas abiertas. La sección siguiente muestra cómo se evidencia su desempeño.

\section{EL PROCESO DE MEDIR LOS ÍCONOS: SIGNIFICADO + DIFERENCIAS}

Como se indica, desplegar información eficiente y efectiva en las etapas de la GRD es fundamental para apoyar la experiencia de las personas. Los símbolos estandarizados o íconos suelen ser una solución, ya que simplifican eficazmente el contenido permitiendo sortear barreras de acceso. El desarrollo de los íconos sigue por lo general un conjunto de reglas aplicables a la forma e implementación, para hacer de este un instrumento reproducible en múltiples soportes. Guemil es un recurso gráfico simple, cuyo diseño considera un acceso y aplicación abierta. El uso inicial ha sido principalmente en plataformas de información (ej. interfaces de usuario). Como se expone a 
continuación, diseñado y disponible el set de íconos, se ha continuado con su medición como herramienta gráfica para validar su desempeño.

\section{Validando el diseño visual: Significado y Diferencias}

Uno de los aspectos cuestionables en los íconos es el ideal de 'universalidad' al que suele aspirar. Esto es, que cada persona pueda interpretar el mismo significado, independiente de su base cultural, idiomática o incluso del momento en que se presenta. Sin embargo, como indica Frascara (2004, p.71): "Un claro ejemplo de la inhabilidad de los diseñadores para evaluar apropiadamente la claridad de un mensaje proviene desde el trabajo realizado por la International Standards Organization (ISO) sobre la comprensión de símbolos gráficos para la información pública. Estos símbolos, los cuales debiesen ser entendidos por personas de diferentes culturas, normalmente muestran un desempeño bastante más bajo de lo que los diseñadores esperan."

Más allá de la declaración de universalidad de los íconos, su desempeño depende de dos variables poco controlables para quien diseña: (1) El contexto en el finalmente que se presenta la información (McDougall et al., 2007) y (2) cómo completa el mensaje una persona, qué interpreta según su base cultural o idiomática. El paso siguiente entonces ha sido diseñar una plataforma paralela para levantar evidencia sobre el desempeño de los íconos. Más allá de su propuesta visual, buscando conocer cómo funcionan los íconos se hizo también necesario conocer qué significados y diferencias pueden hacer los usuarios, evidenciando qué entienden -y qué no- desde las representaciones de la versión $0.5 \mathrm{~b}$ de Guemil.

\section{Metodología aplicada: Cómo se mide el desempeño en significados}

Se desarrollaron algunos principios metodológicos junto a un prototipado experimental de distintos tipos de tests, siguiendo una metodología proyectual y apoyándose en prácticas internacionales relevantes (Olygay, 2001; ISO 2011; ANSI 2011). Así, se seleccionaron 72 de los 96 íconos diseñados para ser incluidos en las pruebas y se estableció medir dos variables cuantificables en su diseño visual: Significado y Diferencias Para levantar indicadores de desempeño se desarrolló un kit de testeo, el que puede accederse desde el sitio web del proyecto. Estos se componen de cinco formas bilingües -inglés y español- empaquetados en archivos pdf interactivos, basadas en distintos conjuntos de preguntas y tareas, que se desglosan en (1) preguntas de respuesta abierta (¿Qué significa este ícono?), (2) elección en base a diferencias (¿Cuál de estos íconos representa ?), (3) relaciones (Asigne un ícono a cada situación), y (4) consideraciones sobre el desempeño (Estos íconos se debieran aplicar en ); ver Figura 8 con ejemplos y respuestas.

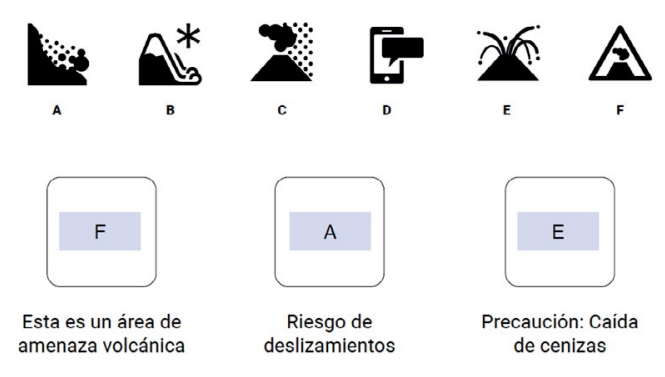

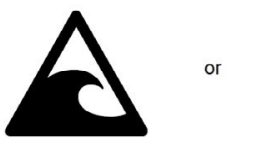

$\square$
Amenaza de Tsunami

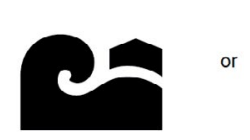

$\checkmark$

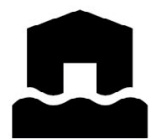

$\square$
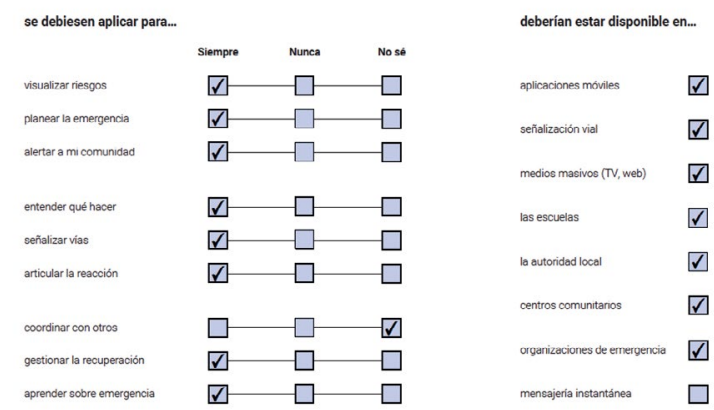
De esta forma, la implementación de los tests fue realizada en base a formularios interactivos con llenado como respuestas abiertas. En el diseño de los de significado que son los que se presentan en este artículo, se buscó una forma de conjugar las preguntas sobre los símbolos y las respuestas abiertas generadas quienes responden, a través de una ponderación de indicadores cuantitativos que indique luego el desempeño de cada ícono. Este índice, expresado en porcentaje es asociado al desempeño de cada ícono define si el ícono es aceptado o no en base a la evaluación de los significados aportados por los usuarios.

Como lo muestra la Figura 9, la evaluación del significado de cada ícono se completa con una ponderación de la respuesta y visualización de su desempeño. Para ello se estableció una tabla de seis valores, basándose en el método que tanto Brugger (1999); Olygay (2003); Frascara, (2011); Boersema \& Adams (2017) sugieren como procedimiento para evaluar la comprensión en símbolos para uso público (ISO, 2007) o para ámbitos asociados a la seguridad en el trabajo (ISO, 2011; ANSI, 2011). Los valores de la escala fueron simplificados, ya que algunos utilizan siete indicadores, separando el valor 6 .

\section{Puntaje Interpretación}

\section{Correcto}

La respuesta es precisa respecto al significado asignado

\section{Casi correcto}

La respuesta es similar o cercana al significado asignado

\section{Dudoso}

La respuesta apunta a otra interpretación o es ambigua

\section{$4 \quad$ Incorrecto}

La respuesta no corresponde al significado asignado

\section{Significado opuesto}

5 La respuesta entregada apunta a un significado contrario

\section{Sin respuesta}

No entrega respuesta o declara no saber el significado

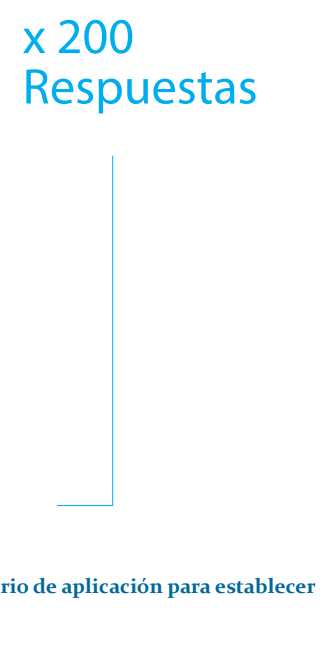

Figura 9: Escala denominada 'Brugger-Frascara' adaptada a seis valores, con el criterio de aplicación para establecer el índice de desempeño de cada ícono Fuente: Autor, 2018

Progresivamente, las respuestas han sido recogidas generando un interesante conjunto de datos, que permite abrir otros campos de análisis. Estas han sido procesadas anónimamente aplicando una serie de herramientas que han permitido pasar desde los formularios en formato pdf a bases de datos, y luego a una plataforma programada para generar visualizaciones, basada en el formato ligero de intercambio de datos conocido como JSON.

Respecto al número (porcentaje) que establece el desempeño de cada ícono, para validar el significado y diferencias en el diseño visual de los íconos se siguieron una serie de reglas. La práctica internacional (ISO, 2011) establece disponer de 200 respuestas válidas por ícono. Otros estándares (ANSI Z353.3, 2011) establecen un rango mínimo de 50 respuestas para validar. De acuerdo a estas mismas prácticas (Frascara 2011; ISO 7010:2011), un ícono aceptado para su utilización como 
estándar de uso público, requiere al menos un $66 \%$ de interpretación cercana a lo que el ícono representa. Sin embargo, para validar su uso en contextos de riesgo o seguridad se establece un mínimo de 83\% para un desempeño aceptable, y estándares como el ANSI Z353.3, 2011 definen incluso un mínimo de $85 \%$.

Así, dependiendo del indicador se considera que los íconos bajo el $83 \%$ podrían ser sometidos a estudio para entender mejor qué factores afectan su significado, antes de considerar su eliminación o re-diseño. Adicionalmente, si se observa más del $5 \%$ en significados opuestos determina que el ícono debiera ser descartado del sistema, dado que su interpretación podría incluso constituir un peligro para quien lo interpreta. La Figura 10 muestra una vista de la plataforma web donde se han expuesto los resultados procesados a septiembre 2017, la que puede verse completa en www. test.guemil.info.

Guemil Project
Sobre el test de desempeño de significados Notas metodolóóicas

La comprensión de cada icono ha sido testeado desde la pregunta: ¿Qué representa cada icono? Siendo respuestas abiertas, cada una ha sido ponderada según una tabla de evaluación.

De acuerdo a prácticas internacionales, un icono aceptado para contextos de emergencia requiere un mínimo de $83 \%$ de desempeño en su significado.

Se considera que los iconos bajo el $83 \%$ de desempeño debieran ser repensados, re-diseñados o descartados. Adicionalmente, más del $5 \%$ en significados opuestos determina descartar y repensar el icono.

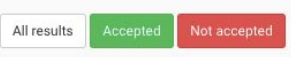

\section{$\pi \stackrel{89 \%}{=}$}

01_Man

Answers Respuestas

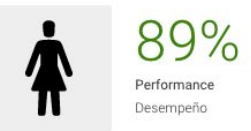

02_Woman
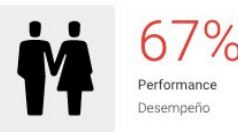

03_Assembly_couple
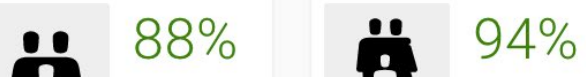

Figura 10: El sitio web test.guemil.info, con el despliegue de resultados de los test de significado (Octubre 2017) Fuente: Autor, 2018

\section{Sobre los resultados del testeo}

Desde los resultados preliminares en la plataforma web se ha habilitado una visualización de los resultados, para su consulta y difusión. Estas permiten visualizar resultados sobre el desempeño de los íconos, y han sido publicados en la web desde mayo 2017, formando parte de la vocación abierta del proyecto. Esta se encuentra en el tramo de alcanzar las 200 respuestas por ícono, obtenidas desde diversos lugares del mundo. Los íconos que sobrepasan el número de respuestas son 43, restando 29 por completar esta cantidad mínima, por lo que los tests se mantienen disponibles para descarga. El procedimiento ha sido también descrito en otras instancias (ver Ramírez, $2016,2017)$ y puede accederse al documento completo con las Methodological Guidelines (en inglés), descargable desde la plataforma GitHub del proyecto.

La visualización de los resultados permite formarse algunas ideas iniciales acerca de la representación basada en símbolos y cómo las personas interpretan en palabras el ámbito de la emergencia, representados en base a elementos que son naturales en su vida cotidiana. Estos son 
aspectos interesantes como referencia de conocimiento en diseño y para la investigación basada en proyectos. La colaboración y divulgación de distintas organizaciones ha resultado importante para promoverlo (CIGIDEN, 2016-2017; DNEM, 2016). La Figura 11 presenta una visualización extendida con el desempeño de los íconos tras el testeo de significados. Este conjunto ha sido dispuesto aleatoriamente para mostrar el desempeño, junto a la cantidad de respuestas.

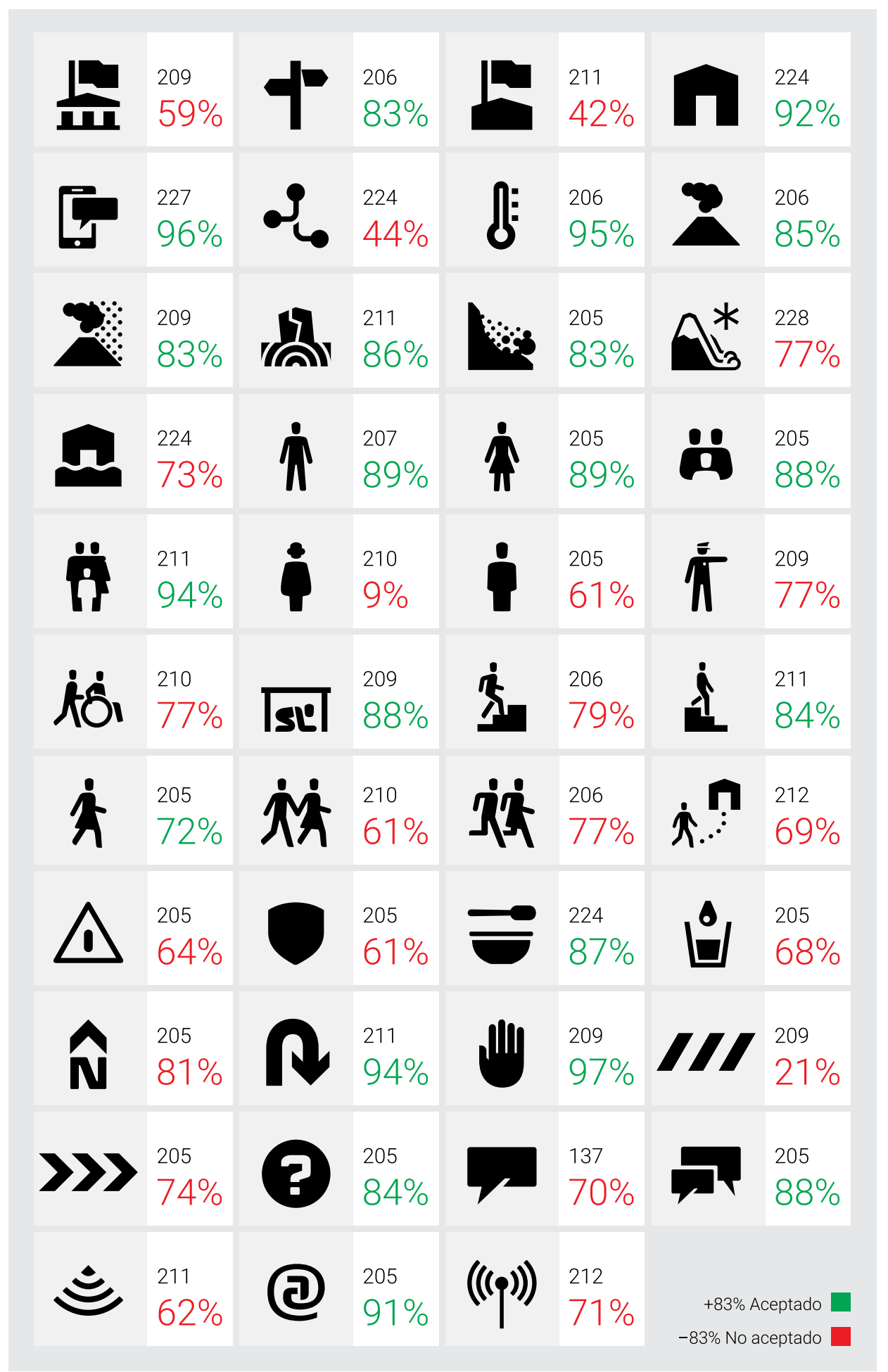

Figura 11: Visualización simplificada de los íconos con más de 200 respuestas con índice del desempeño, a Octubre 2017 Fuente: Autor, 2018 
Como observaciones generales sobre la filiación en los resultados, puede mencionarse la predominancia de respuestas locales (Chile) y de la región latinoamericana, siendo una tarea pendiente una mayor difusión internacional. También, dado que las respuestas han sido recogidas en gran parte en ámbitos académicos, se observa en estas una desviación por rango etáreo (16-35 años), y también por idioma (español). Un hecho que llama la atención es la incidencia marcada de quienes responden declaran 'estar familiarizados con la emergencia'. Para graficar en detalle las respuestas que los usuarios dan a los íconos, la Figura 12 presenta a continuación cuatro casos.

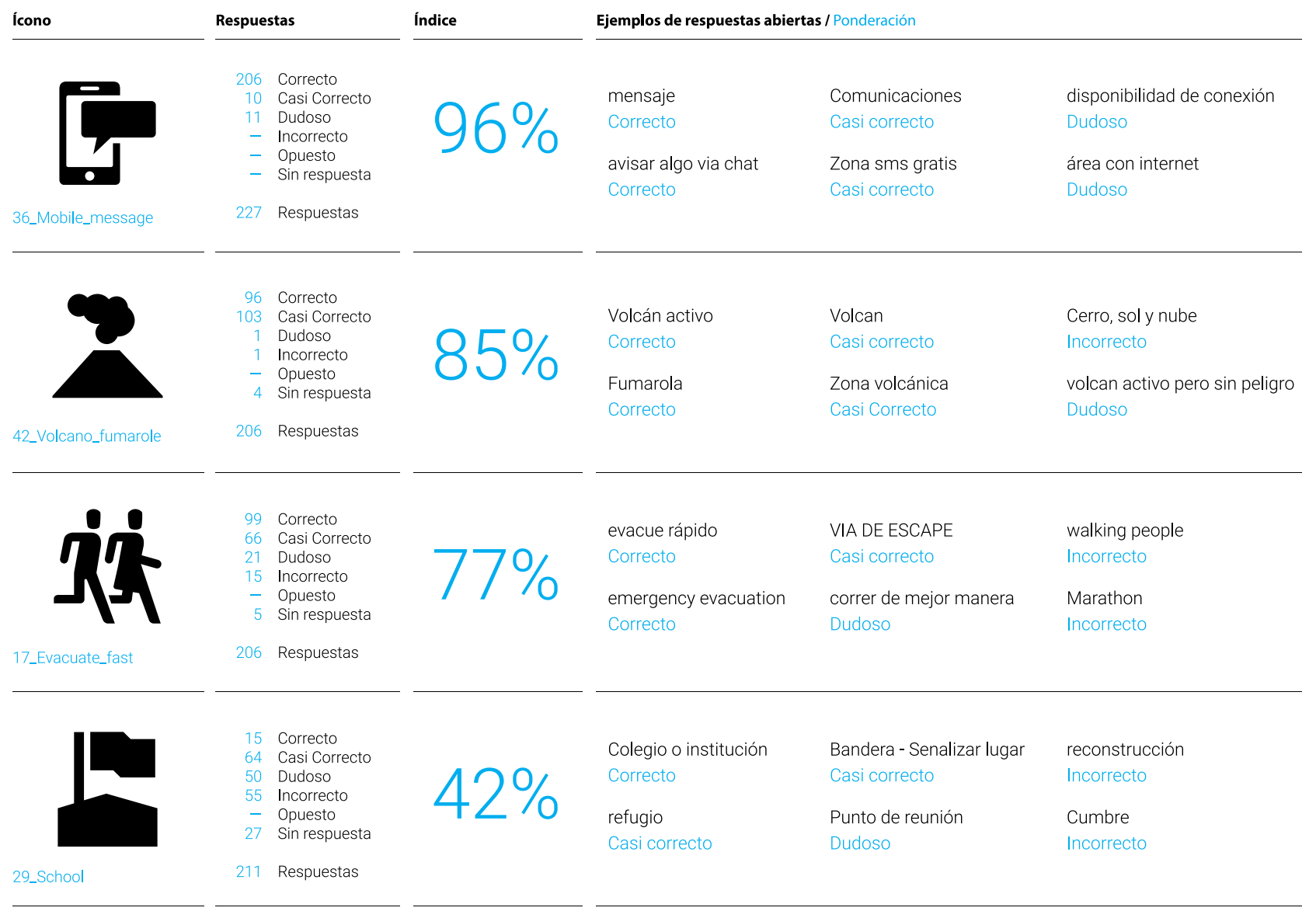

Figura 12: Visualización de aspectos específicos de significados en 4 íconos Guemil: Respuestas abiertas y ponderación respecto al desempeño: 1. Óptimo desempeño (sobre 90\%); 2. Aceptado (sobre 83\%); 3. No aceptado (bajo 83\%); 4. Bajo desempeño (bajo 66\%).

Fuente: Autor, 2018

Aunque se encuentra todavía en vías de completarse, el número de respuestas recogidas y la difusión internacional del proyecto muestran el interés que este tipo de iniciativas puede llegar a tener globalmente.

Como se muestra existen proyecciones interesantes tanto para el proceso de diseño como para la validación y análisis de los resultados del testeo. Un proyecto que es al mismo tiempo un proceso de investigación contribuye a abrir el debate y surgen nuevos ejemplos de los que se puede hacer con una información diseñada y la evidencia que genera. También es posible analizar los significados asociados a la emergencia para definir qué se puede mejorar: pertinencia, lenguaje técnico, representación. Desde la experiencia de interpretación y conjunto de datos, se estiman dos tareas para la continuidad: [1] Revisar los íconos en cuanto a significados y cantidad, optimizando las diferencias. [2] Explorar nuevos indicadores, para generar evidencia sobre el desempeño de la información gráfica en contextos de riesgo, siempre con una mirada en la colaboración. Desde los resultados del proyecto en proceso, se señalan también algunas reflexiones en la sección siguiente. 


\section{PROYECTANDO UNA COMUNICACIÓN EFECTIVA DEL RIESGO}

Una tarea sencilla como responder sobre significados y diferencias puede ser de ayuda para conocer y medir qué es lo que los ciudadanos entienden y retienen sobre la preparación, sobre conductas de riesgo, o cuando un desastre se ha desencadenado. Tanto como proyecto de símbolos y como proceso de investigación se considera que los resultados del proyecto permiten mirar hacia adelante, promoviendo su continuidad. Siendo un caso en proceso, algunas de las variables podrían sumar análisis específicos desde otras disciplinas en el tiempo, ampliándose a trabajar desde las ciencias, la psicología o la educación, con evidencia disponible para desarrollos a posterior. Enfocadas desde lo metodológico a acciones prácticas, se discuten a continuación nociones sobre la comunicación de información en la emergencia, reflexionando sobre el eventual impacto que herramientas efectivas pueden aportar a las personas.

\section{Proyectar metodologías de diseño a otras disciplinas}

La experiencia de Guemil muestra un proceso creativo, que trabaja desde el diseño en un ámbito prioritario, hacia un diálogo posible con múltiples disciplinas. Es clave su enfoque centrado en la experiencia de las personas, ya que genera nuevas perspectivas, permite visualizar otros escenarios y entender las múltiples interpretaciones que la comunicación del riesgo pueda presentar. Metodologías como las que emplea el diseño pueden contribuir a aspectos fundamentales en la GRD, explorando otros escenarios, perfilando quiénes forman parte y desarrollando nuevas aproximaciones.

Independiente de qué métodos se apliquen, es posible proyectar el enfoque clásico del diseño -creación + función- a través de un ciclo de acciones fundamentales: Explorar > Prototipar > Testear. Este puede ir desde detectar necesidades no cubiertas, entender qué es significativo en la preparación, hasta factores culturales asociados a la recuperación. Tal vez una de las proyecciones más interesantes del proyecto Guemil sea la de insertarse en escenarios alternativos, donde explorando nuevos soportes de información se recoja evidencia que optimice la comunicación del riesgo.

De esta forma, metodologías como las que se emplean en diseño pueden contribuir a la colaboración, a centrarse en aspectos significativos y en empoderar a las organizaciones. Refinando su aplicación, testeos como los aplicados en el proyecto Guemil podrían contribuir a identificar percepciones sobre qué funcionaría mejor al desplegar otros tipos de información en contextos críticos, con una iteración continua en el tiempo.

\section{Promover una preparación centrada en el aprendizaje: qué y cómo hacer}

Un aspecto importante en las políticas de GRD es cómo articular acciones eficientes: Una clave sería facilitar el aprendizaje entregando herramientas para llevarlas a cabo. Como parte de un proceso de diseño, conocer qué información requieren las comunidades y prototipar escenarios de preparación, contribuiría al aprendizaje basado en qué hacer -y qué no-. Más allá, se puede también promover el aprendizaje sobre cómo hacer, desplegando información instruccional simple y consistente.

Siendo la información un soporte estructural para las etapas de la GRD, la plataforma web de Guemil dispone hoy un insumo base para generar elementos de información que faciliten este aprendizaje. Soluciones de este tipo se han desarrollado con éxito en lugares como Japón, donde su distribución es pública. Futuros desarrollos podrían avanzar hacia plataformas que permitan a las personas integrar qué y cómo hacer ante la emergencia de forma práctica, evitando esperar a enfrentarse a un momento crítico con incertidumbre.

El desarrollo futuro de Guemil podría constituir un sistema integral, que permita desplegar información oportuna que visibilice riesgos y contribuya entre otras cosas a manejar la desinformación o incluso a promover la resiliencia, en ciclos de preparación que aborden las distintas etapas. Por ejemplo, generar soluciones multi-escenario que compartan un mismo estándar gráfico (familiar a las personas). Si estas se aplican para visualizar otras amenazas, podría permitir sumar el manejo anterior sobre qué y cómo proceder con un lenguaje unitario. A largo plazo podrían abordarse otras necesidades de información, siempre centrados en contribuir a la experiencia de las personas. 


\section{Adoptar los íconos para consolidar el proyecto}

Los escenarios asociados al riesgo de desastres son permanentes y siempre críticos en cuanto a la demanda de información. Por lo tanto, la aplicación de herramientas que permitan lidiar con estos también debiera serlo. Un proceso de diseño puede extenderse en una iteración constante donde diversos equipos colaboren para optimizar su resultado. Ello es clave para consolidar iniciativas de comunicación del riesgo como Guemil.

Uniendo las necesidades de información con un producto visual, Guemil es un proyecto donde la investigación y el desarrollo han permitido generar un resultado usable, ante un desafío que presenta evidentes demandas y espacios para aplicarlo. Es clave que un recurso abierto, que normaliza una información que puede ser de beneficio público sea considerado como recurso adoptable. Otra oportunidad abierta para la colaboración, ya que los íconos Guemil podrían también validarse a partir de su adopción por organizaciones que lideran la gestión del riesgo y la emergencia (ej. gobiernos). Este debiese ser un próximo paso para una mejora y consolidación.

También, esta adopción podría facilitar el desarrollo de nuevas soluciones de comunicación para la preparación, mitigación y recuperación de desastres de mayor envergadura, aunque compartiendo un mismo estándar gráfico, escalable a nivel local, regional, global. Por lo tanto, adoptarlo por parte de organizaciones relacionadas permitiría mejorarlo colaborativa y continuamente lo que sería útil al promover los íconos con mejor resultado. La medición del desempeño en los significados es una forma de evidenciar indicadores, donde cada ícono disponible podría mejorarse desde índices abiertos para su evaluación.

\section{CONCLUSIÓN}

Un atributo central del diseño es trabajar en torno a la experiencia de las personas. En un contexto crítico como el de la emergencia, esta experiencia se altera y se hace necesario contar con información adecuada y oportuna. Los enfoques del diseño centrados en las personas pueden ayudar a entender las necesidades y conformar soluciones que podrían optimizar la comunicación, impactando significativamente en la GRD. Específicamente, el caso de los íconos Guemil demuestra que es posible conducir iniciativas combinando la experiencia de usuario y este ciclo, para facilitar el acceso a través de elementos gráficos simples, que permitan visibilizar, entender y traducir la información a acciones.

En su progresión, el proyecto Guemil emerge como un insumo base para el desarrollo de mensajes visuales; una solución simple con un lenguaje normalizado. Su testeo ha permitido generar evidencia sobre los significados reconocidos, dando cuenta de cómo estos son definidos por los usuarios al completar un mensaje y qué diferencias establecen, mostrando su desempeño. Es importante considerar que herramientas de información consistentes, con mediciones centradas en lo que las personas comprenden, pueden contribuir a optimizar la experiencia y a hacer más eficiente la comunicación del riesgo. Por ello se plantea avanzar en promover su adopción. Se puede escalar hacia otras implementaciones y a acciones que faciliten el aprendizaje, conformando por lo tanto oportunidades de enriquecerlo.

Por último, escenarios futuros plantean desafíos donde se hace necesaria la integración y colaboración disciplinar. Esta es una oportunidad de promover transformaciones articuladas por dinámicas como los que se plantean desde el diseño donde se explore, se desarrolle y se evalúen las soluciones de forma permanente. En este sentido, el foco sobre el aprendizaje para la preparación es fundamental. Los íconos como lenguaje de familiarización, pueden facilitar acciones como la preparación y sus procedimientos.

\section{AGRADECIMIENTOS}

Al equipo de trabajo que ha contribuido al desarrollo del proyecto: Felipe Cortez, Francisca Balbontín, Laura Mena, Felipe Vilches, Denisse Ortega. Al board del Design Network for Emergency Management (dnem. org). También al soporte entregado por la Escuela de Diseño de la Pontificia Universidad Católica de Chile, al Centro de Investigación para la Gestión Integrada de Desastres, CIGIDEN, y a School of Design, The Hong Kong Polytechnic University. 


\section{REFERENCIAS}

Abdullah, R. \& Hübner, R. (2006). Pictograms, Icons, and Signs. London: Thames \& Hudson.

ANSI Z353: American National Standard, Criteria for Safety Symbols (preview document), recuperado desde: https://webstore.ansi.org/Previews/PREVIEW_ANSI+NEMA+Z535.3-2011.pdf

Boersema, T. \& Adams, A. (2017). Does my symbol sign work?: International standards for designing and testing graphical symbols. In Black, A.; Luna, P. et al., eds. Information Design Research and Practice. London: Routledge.

Brugger Ch. (1990). Advances in the international standardization of public information symbols. Information Design Journal, 6(1), pp.79-88.

Brugger, Ch. (1999). Public Information symbols: a comparison of ISO testing procedures. Visual Information for Everyday Use. London: Taylor \& Francis.

Bui, T. \& Sebastian, I. (2011). Beyond Rationality: Information Design for Supporting Emergent Groups in Emergency Response. In Burstein, B., \& Zaslavsky, E. (Eds.), Supporting Real Time DecisionMaking, New York: Springer.

Cross, N. (2001). Designerly ways of knowing: design discipline versus design science. Design Issues, 17(3) pp.49-55.

Environment Agency, UK. (2018) Environment Agency. Recuperado el 14 Feb. 2018 desde: www.environment-agency.gov.uk

First Aid Icon Initiative. (2018). Project. Recuperado el 14 Feb. 2018 desde: www.buerobauer.com

Flood Warnings Information Service. (2018). Recuperado el 14 Feb. 2018 desde: https://flood-warning-information.service.gov.uk/warnings

Frascara, J. (2004). Communication Design: Principles, Methods and Practice. New York: Allworth Press.

Frascara, J. (2011). Qué es el Diseño de Información. Buenos Aires: Ed. Infinito.

Frascara, J. (2015). Information Design as Principled Action: Making Information Accessible, Relevant, Understandable, and Usable. Champaign, IL: Common Ground Research Networks.

Github - Guemil. (2018). Guemil Project. Recuperado el 14 Feb. 2018 desde: https://github.com/Guemil/ Guemil_Project

Guemil Project website. (2018). Recuperado el 14 Feb. 2018 desde: http://guemil.info

Horn, R. (1999). Information Design: Emergence of a New Profession. In Jacobson, R. (Ed.). Information Design. Cambridge: MIT Press, pp.15-33.

Isherwood, S., McDougall, S., \& Curry M. (2007). Icon Identification in Context: The Changing Role of Icon Characteristics with User Experience. Human Factors, 49(3). DOI: 10.1518/001872007X200102

ISO. (2009). Ergonomics of human system interaction - Part 210: Human-centered design for interactive systems. Recuperado el 14 Feb. 2018 desde: https://www.iso.org/standard/52938.html

ISO. (2011). ISO 7010:2011, 3.7 - Graphical symbols -- Safety colours and safety signs -- Registered safety signs. Recuperado el 14 Feb. 2018 desde: http://www.iso.org/iso/home/store/catalogue_tc/catalogue_detail.htm?csnumber $=54432$

Jaenichen, C. (2011). Design for Evacuation: Improving City Evacuation Instructions. Parsons Journal for Information Mapping, 3(2), pp.1-16.

Mijksenaar, P. \& Westendorp, P. (2001). Open Here: The Art of Instructional Design. London: Thames \& Hudson.

Anónimo/a. (2015). Disaster Preparedness Tokyo Bousai [PDF]. Recuperado el 14 Feb. 2018 desde: http://www.metro.tokyo.jp/ENGLISH/GUIDE/BOSAI/index.htm

Norman, D. (2004). Emotional Design. New York: Basic Books.

Olgyay, N. (2001). Development \& testing of the IIID safety symbols system. Information Design Journal, 10(2). DOI: 10.1075/idj.10.2.05olg

Olygay, N. (2003). Building on test Results to design Safety Symbols. Proceedings, XV Triennial Congress of the International Ergonomics Association in Seoul, Korea.

Parsons, S., Seminara, J. \& Wogalter, M. (1999). A Summary of Warnings Research. Ergonomics in Design, 7 (1), pp.21-31. DOI: 10.1177/106480469900700105

Pettersson, R. (2002). Information design, an introduction. Amsterdam: John Benjamins Publishing. 
Ramírez, R. (2017). Guemil: Diseño y medición de significados de un set de íconos para representar el riesgo y la emergencia. Manuscrito presentado en el Congreso SIGRADI, Chile.

Ramírez, R. (2017). Information Design for Emergency [PDF]. Recuperado el 14 Feb. 2018 desde: https:// www.academia.edu/33867534/Lecture_3_Information_Design_for_Emergency

Ramírez, R. (2017). Reviewing Open-access Icons for Emergency: A Case Study Testing Meaning Performance in Guemil. Trabajo no publicado.

Ramírez, R. \& Ramírez, S. (2014). Notas Sobre el diseño de un sistema de Información Visual. En Allard, J., Briones, M., Gálvez, F., Ramírez R., Ramírez, S. \& Zurob, C. (Eds.) El diseño que pasa inadvertido. Santiago: Pontificia Universidad Católica de Chile, Escuela de Diseño.

Tokio Bousai Disaster Prevention website. (2018). Tokio Bousai Disaster Prevention. Recuperado el 14 Feb. 2018 desde: http://www.bousai.metro.tokyo.jp/foreign/english/index.html

TorqAid Disaster Risk Management Diagrammatic Framework. (2016). TorqAid. Recuperado el 14 Feb. 2018 desde: http://www.torqaid.com/resources/

UNISDR. (2005). Marco de Acción de Hyogo para 2005-2015: Aumento de la resiliencia de las naciones y las comunidades ante los desastres. Kobe: UNISDR.

UNISDR. (2015). Marco de Acción de Sendai para 2015-2025. Sendai: UNISDR.

UNISDR. (2015). UNISDR Terminology. Recuperado el 14 Feb. 2018 desde: https://www.unisdr.org/we/ inform/terminology

Wasson C. (2000). Ethnography in the Field of Design. Human Organization, 59(4), pp.377-388.

Wogalter, M. (1999). Factors influencing the effectiveness of warnings. En Zwaga, H, Boersema, T. \& Hoonhut, M. (Eds), Visual Information for everyday use. London: Taylor \& Francis.

Zender, M. \& Cassedy, A. (2014). (mis)Understanding icon comprehension in different cultural contexts. Visible Language, $48(1)$.

Zender, M. \& Mejía, M. (2013). Improving Icon Design: Through Focus on the Role of Individual Symbols in the Construction of Meaning. Visible Language, $47(1)$.

Zwaga, H. \& Mijksenaar, P. (200o). The development and standardization of Warning Symbols; The role of Design and Human Factors. Proceedings of the Human Factors and Ergonomics Society Annual Metting, USA. 